\section{Outcome of complete intrastromal ring implantation using femtosecond laser in pellucid marginal degeneration}

M Jabbarvand, H Hashemian, M Khodaparast, $\mathrm{F}$ Bazvand and A Beheshtnejad

\begin{abstract}
Purpose To evaluate the effect of complete intrastromal corneal ring implantations on patients with pellucid marginal degeneration (PMD).

Design Prospective interventional case series Patients and Methods Thirty-three eyes with PMD were included into the study. After pocket creation with femtosecond laser (Femtec; 20/10 PerfectVision), MyoRing implantation was performed. Uncorrected and corrected distance visual acuity (UDVA, CDVA), subjective refraction, keratometry, central corneal thickness, corneal biomechanical profile (Ocular Response Analysis), and whole-eye wavefront aberrometry (iTrace) were evaluated preoperatively and also postoperatively, 1 month, 3 months, 6 months, and 1 year after the operation.
\end{abstract}

Results One month after surgery, significant improvements were observed in UDVA (ANOVA; $P=0.02)$, mean keratometry, sphere (ANOVA; P < 0.001), and cylinder (ANOVA; $P=0.04)$ with no significant changes afterwards. No significant change occurred in the corneal biomechanical profile. Primary coma and trefoil reduced after 1 year (ANOVA; $P$ values were 0.02 and 0.06 , respectively). Primary spherical aberration significantly increased according to the 1-year follow-up (ANOVA; $P<0.001$ ). No significant complication was observed.

Conclusion MyoRing is considered as a treatment modality for spherocylindrical correction in patients with PMD, with an acceptable safety and efficacy profile.

Eye (2015) 29, 783-790; doi:10.1038/eye.2015.33; published online 27 March 2015

\section{Introduction}

Pellucid marginal degeneration (PMD) is characterized by non-inflammatory corneal thinning being more prominent in the inferior part of cornea in a crescentic shape with protrusion above the area of thinning. This altered corneal shape leads to against-the-rule astigmatism and visual acuity deterioration. ${ }^{1-6}$ The etiology is unknown. ${ }^{7}$ Different treatment modalities are utilized to manage the patients with PMD based on the severity of their disease, including spectacle, rigid gas permeable contact lens, intrastromal corneal ring segments (Intacs, KeraRing), and several other surgical techniques. ${ }^{1,8,9}$

Intracorneal ring segment (ICRS) is considered an effective, safe, and reversible treatment modality in the vision rehabilitation of patients with corneal ectatic disorders. Inserting these implants in the mid-periphery of the cornea remodels corneal curvature and transforms a protruded thin cornea into a more regular shape. These changes lead to vision improvement and more stable refraction, and also reduce the higher order aberrations. The arc shortening effect of these segments is directly related to their thickness. ${ }^{10,11}$

Application of Intacs (Addition Technology) and KeraRing (Mediphacos, Belo Horizonte, Brazil) in PMD treatment is reported to be safe and effective. 1,9 Safety and efficacy of newly proposed complete intrastromal ring (Myoring, DIOPTEX GmBH, Linz, Austria) have been proved in keratoconus cases. ${ }^{12-14}$ There is no previous report of MyoRing implantation for patients with PMD. Despite the previously reported equal outcomes for ICRS, the newly proposed femtosecond laser seems to act more accurately in comparison with the manual techniques in pocket creation. ${ }^{15-17}$ Clinical results of MyoRing implantation in a group of patients with PMD are reported here.
Ophthalmology Research Center, Farabi Eye Hospital, Tehran University of Medical Sciences, Tehran, Iran

Correspondence:

H Hashemian, Eye Research Center, Farabi Eye Hospital, South Kargar St, Tehran, Iran

Tel: +98 912680 5097;

Fax: +98 2155409095

E-mail: h-hashemian@

tums.ac.ir

Received: 21 April 2014 Accepted in revised form: 1 February 2015 Published online: 27 March 2015

The article has been presented as an oral presentation at the ASCRS meeting 2012. 


\section{Patients and methods}

The current prospective, interventional case series was conducted in Farabi Eye Hospital. One surgeon (MJ) went through all procedures. Informed consent was obtained from all patients, after presenting other treatment options. Institutional ethical review board approval was obtained for the procedures, and the tenets of the Helsinki Declaration were followed. Patients with PMD were included in this study. The diagnosis was made according to slit-lamp examination (ectasia above the area of maximum thinning in the inferior cornea), corneal topography ('butterfly' keratometry pattern, very steep peripheral inferior cornea, and high keratometric powers radiating from the center to the inferior oblique meridians), and refractive findings (against-the-rule astigmatism with loss of corrected distance visual acuity (CDVA)). ${ }^{18}$

Procedure safety was defined as the percentage of eyes having lost more than 2 lines of Snellen CDVA. ${ }^{8}$ The safety index was calculated by dividing the mean postoperative CDVA by the mean preoperative CDVA. ${ }^{8,9}$ Efficacy of a refractive procedure was defined as the percentage of operated eyes achieving 20/40 UDVA or more. ${ }^{9}$ The efficacy index was calculated by dividing the mean postoperative UDVA by the mean preoperative CDVA. And, stability index was the percentage of eyes with less than $1 \mathrm{D}$ change in spherical equivalent between the first to twelfth postoperative months. ${ }^{9}$

In our practice, we considered some contra-indications for MyoRing implantation including glasses tolerance with CDVA of higher than 20/30, contact lens tolerance, central corneal opacity, mesopic pupil diameter of above $5 \mathrm{~mm}$ (according to Orbscan report in mesopic conditions), other ocular pathologies (eg, cataract, glaucoma, and diabetic retinopathy), plano or hyperopic spherical equivalent, history of herpes keratitis, systemic connective tissue disease, pregnancy, and minimum corneal thickness $360 \mu \mathrm{m}$ or less. In addition, we excluded patients with previous ocular surgery from this study.

Patients' visual acuity was evaluated by Snellen chart and converted to logMAR to be analyzed. All patients were examined preoperatively and also within 1 month, 3 months, 6 months, and 1 year after the surgery. During each visit, complete ocular examination was performed, consisting of UDVA and CDVA evaluation, refraction, slit lamp biomicroscopy, Goldman applanation tonometry, and fundoscopy. Ultrasonic pachymetry (SP3000; Tomey, Nagoya, Japan), Pentacam corneal imaging (Oculus Optikgeräte GmbH, Wetzlar, Germany), Ocular Response Analysis (ORA; Reichert, Buffalo, NY, USA), and wholeeye aberrometry (Tracey Technologies, Houston, TX, USA) were also carried out in all visits.

\section{Surgery method}

A stromal pocket was produced by femtosecond laser (Femtec; 20/10 PerfectVision; GmbH, Heidelberg, Germany) in all cases. This solid-body pulsed Nd:glass laser has a repetition rate of $40 \mathrm{kHz}$, and a wavelength of $1059 \mathrm{~nm}$ with a pulse duration of $600-800 \mathrm{fs}$. For this procedure, energy level of $1.05 \mu \mathrm{J}$, spot size of $5 \mu \mathrm{m}$, spot spacing of $3 \mu \mathrm{m}$, and line spacing of $5 \mu \mathrm{m}$ were chosen. The provided contact lens is concave and curved with a 10.5 -mm radius of curvature. First, the pupil center was marked with a Sinskey hook before applanation. This mark was used as a reference point to locate the incision and to center the MyoRing after implantation. A temporal corneal incision of 70 degrees of arc length was made, and afterwards an intrastromal pocket of $9 \mathrm{~mm}$ in diameter and $300 \mu \mathrm{m}$ in depth was created. Once the pocket was created, the MyoRing (DIOPTEX GmBH) was inserted into the pocket (Figure 1a). We used our adapted nomogram to calculate the MyoRing size (Table 1). The surgery was performed under topical anesthesia. Subsequently, the bandage contact lens with no sutures was applied. Topical Chloramphenicol drops were administered every $6 \mathrm{~h}$ for 1 week and topical lubricant and Betamethasone were used for 1 month.

\section{Vector analysis}

The astigmatic correction effect and accuracy was calculated using Alpins vector analysis method. ${ }^{19}$ Postoperative refractive astigmatism was compared with preoperative astigmatism. As emmetropia was the goal in all patients, target point of astigmatism was zero. Magnitude (diopters) and axis (degrees) of astigmatism were transformed into rectangular $x$ and $y$ coordinates to calculate the following variables and vectors. The surgically induced astigmatism (SIA), target-induced astigmatism (TIA), difference vector and its angle, index of success (IOS), correction index, flattening effect, and flattening index were calculated.

IOS is a measure of success based on the goal for astigmatic correction. Zero value is desirable and indicates that the astigmatism is fully corrected (zero astigmatism). The value can be between 0 and 1 , for example, an index of 0.5 indicates $50 \%$ success in astigmatic correction. If it equals 1 , then surgery has changed the astigmatism to a point being equally far away from the target point as the preoperative value. Values higher than 1.0 indicate worse results than preoperative astigmatism. The TIA is a vector representing the desired change (by magnitude and axis) the operation was intended to induce. The SIA is the vector of actual change induced by the surgery. The difference vector (DV) is the treatment vector needed to 
a

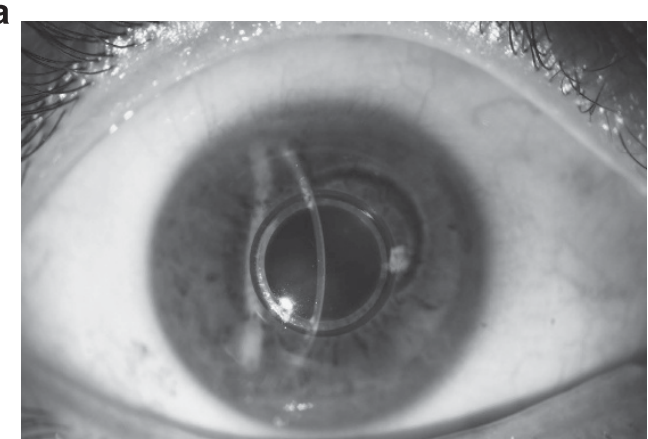

b

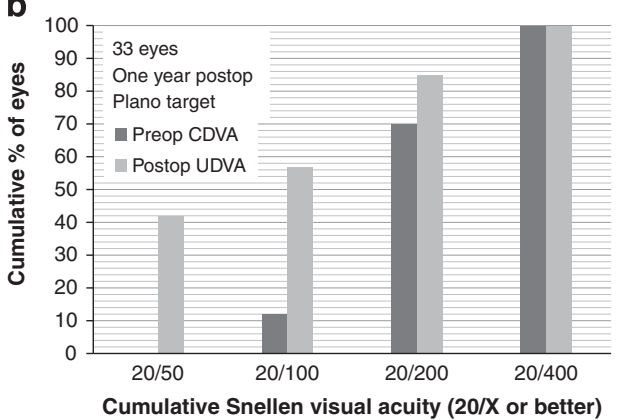

d
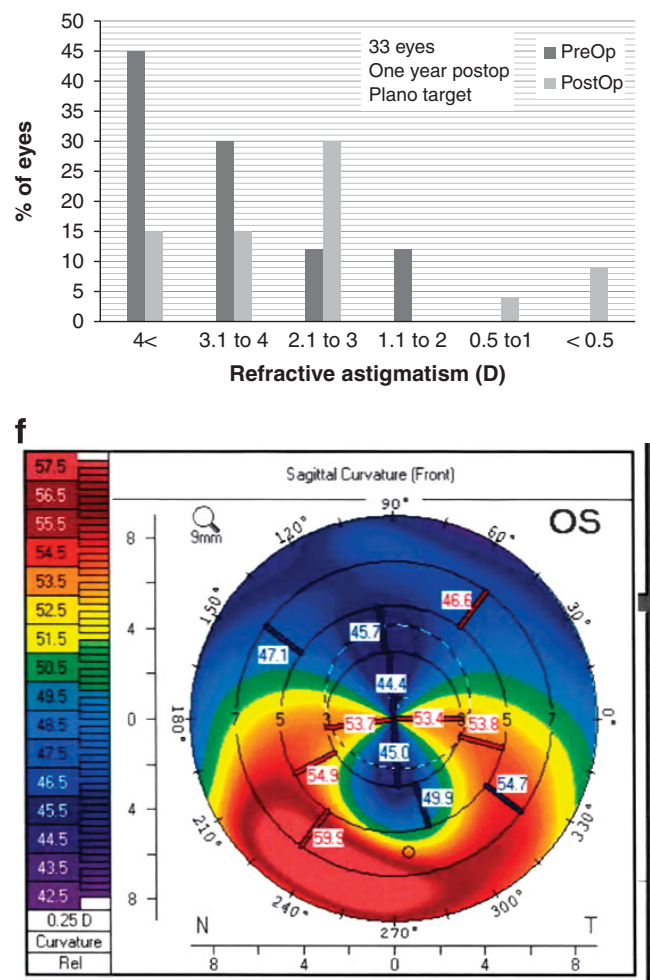

C

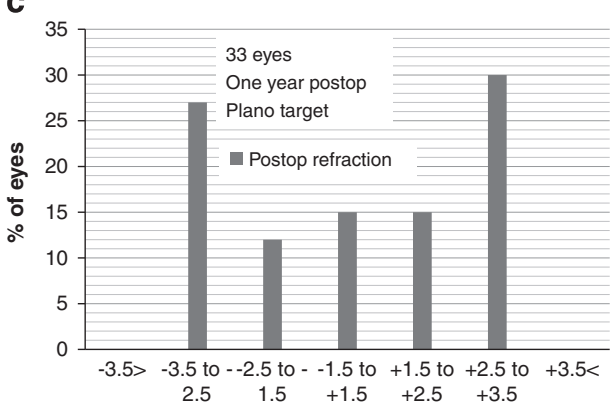

Postoperative spherical equivalent refraction (D)
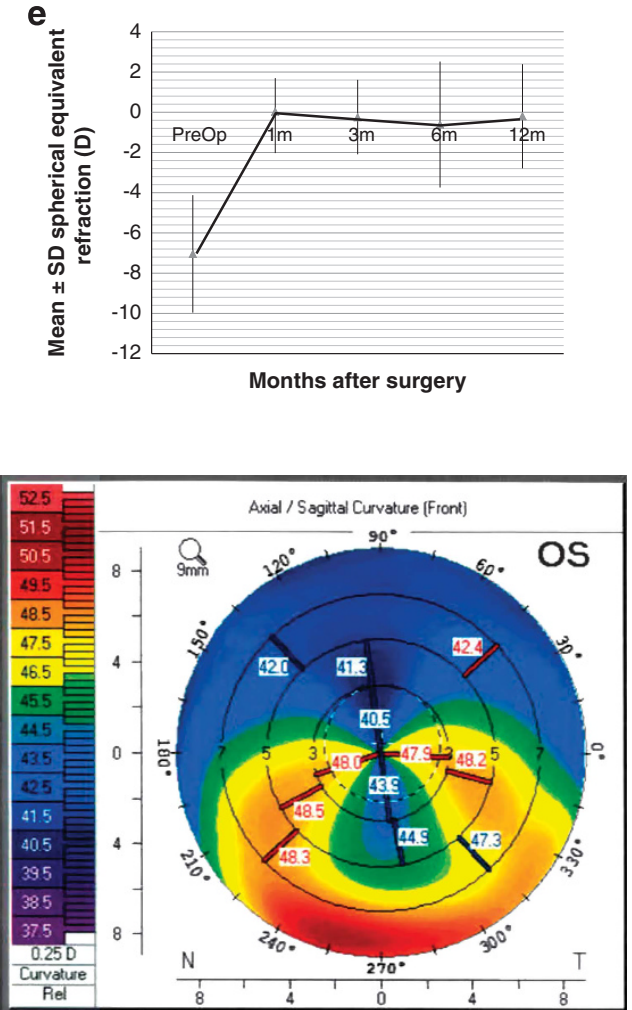

Figure 1 (a) Slit photograph of MyoRing implanted in corneal pocket. (b) Bar graph of cumulative percentage of preoperative CDVA against postoperative UDVA. (c) Final residual refraction (spherical equivalent). (d) Final refractive astigmatism. (e) Changes of spherical equivalent refraction preoperatively and during the follow-up period. (f) Keratometric map of a patient with PMD before (left) and 6 month after (right) MyoRing implantation. 
achieve the target goal after the initial surgery. This is also a measure of success, which preferably equals zero. The correction index was calculated by dividing SIA to TIA. The correction index is preferably 1.0. This index would be higher than 1.0 in case of overcorrection and lower than 1.0 if undercorrection occurs. The angle of error is the angle between the SIA and TIA vectors. The angle of error is positive if the achieved correction is counterclockwise to the intended astigmatism axis and negative if the achieved correction is clockwise to the intended axis. The flattening effect is the reduction in astigmatism value induced by the proportion of SIA, which is effective at the intended meridian (flattening effect $=$ SIA $\cos 2 \times$ angle of error). The flattening index is obtained by dividing the flattening effect by the TIA, which preferably equals 1 .

\section{Statistical analysis}

The Kolmogorov-Smirnov normality test was used to test the normal distribution of continuous variables. Variables

Table 1 Our modified nomogram for corneal intrastromal ring implantation

\begin{tabular}{llcc}
\hline K value & Mesopic pupil & \multicolumn{2}{c}{ Ring dimension } \\
\cline { 3 - 4 } & & Diameter $(\mathrm{mm})$ & Thickness $(\mu \mathrm{m})$ \\
\hline $\mathrm{K} \leq 44$ & $<5.5^{\mathrm{mm}}$ & 6 & 240 \\
$44<\mathrm{k} \leq 48$ & $<4.5^{\mathrm{mm}}$ & 5 & 240 \\
& $\geq 4.5^{\mathrm{mm}}$ & 6 & 280 \\
$48<\mathrm{k} \leq 52$ & $<4.5^{\mathrm{mm}}$ & 5 & 280 \\
& $\geq 4.5^{\mathrm{mm}}$ & 6 & 320 \\
$\mathrm{~K}>52$ & $<4.5^{\mathrm{mm}}$ & 5 & 320 \\
\hline
\end{tabular}

Abbreviation: K, Keratometry. with and without normal distribution were compared using one-way ANOVA and Kruskal-Wallis variance analysis, respectively. Scheffe test was used for post hoc test. Comparison of parameters with fixed numbers in vector analysis was performed using one sample $T$ test. The $P$ values of less than 0.05 were considered statistically significant. Calculations were made using statistical analysis software (IBM SPSS Statistics 16; SPSS Inc, Chicago, IL, USA).

\section{Results}

Thirty-three eyes (19 right eyes and 14 left eyes) of 33 patients were included in this investigation. The mean age of the patients was 29 years (range: 21-43 years). Eighteen patients were male $(54 \%)$.

Clinical results of the patients are shown in Table 2. UDVA improvement was significant 1 month after the surgery $(P=0.02)$. However, no statistically significant further improvement was found in any postoperative time point $(P$ values $<0.05)$. Contrariwise, CDVA did not change significantly after the operation $(P=0.6)$. It did not change significantly even after the 12-month follow-up period. $(P=0.06)$ The reduction in spherical and cylindrical parts of refraction was significant 1 month $(P<0.001$ and $P=0.04$, respectively) after the surgery without any additional reduction afterwards. (Figures $1 \mathrm{~b}-\mathrm{e}$ ) A significant reduction in mean keratometry was observed 1 month after the surgery $(P<0.001$, Figure 1f). However, there was no further change afterwards during the 1-year post-operative period $(P=0.95)$. Central corneal thickness did not change significantly in any time point after the operation. Corneal biomechanical profile (corneal resistance factor and corneal hysteresis ) had no

Table 2 Summary of visual, refractive, aberrometric, and biomechanical outcomes before corneal intrastromal ring implantation for treatment of keratoconus and during the postoperative 1 year follow-up period

\begin{tabular}{|c|c|c|c|c|c|c|c|}
\hline & \multirow[t]{2}{*}{ Preop. } & \multicolumn{4}{|c|}{ Post operation } & \multicolumn{2}{|c|}{ P-value } \\
\hline & & $1 m$ & $3 m$ & $6 m$ & $12 m$ & Pre-12m & $1-12 m$ \\
\hline UDVA & $1.11 \pm 0.24$ & $0.75 \pm 0.37$ & $0.65 \pm 0.27$ & $0.62 \pm 0.30$ & $0.62 \pm 0.34$ & 0.003 & 0.9 \\
\hline CDVA & $0.68 \pm 0.18$ & $0.60 \pm 0.15$ & $0.56 \pm 0.09$ & $0.50 \pm 0.08$ & $0.50 \pm 0.17$ & 0.06 & 0.6 \\
\hline Sphere & $-4.46 \pm 2.28$ & $1.00 \pm 1.54$ & $0.82 \pm 1.68$ & $0.45 \pm 1.84$ & $1.02 \pm 1.96$ & $<0.001$ & 0.8 \\
\hline Cylinder & $-4.78 \pm 2.34$ & $-1.71 \pm 1.70$ & $-2.21 \pm 1.64$ & $-1.85 \pm 0.91$ & $-2.07 \pm 2.23$ & 0.002 & 0.4 \\
\hline Mean Keratometry & $52.32 \pm 3.79$ & $45.25 \pm 3.36$ & $45.44 \pm 3.50$ & $45.14 \pm 4.23$ & $45.22 \pm 3.62$ & $<0.001$ & 0.95 \\
\hline CCT & $430.7 \pm 23$ & $448.8 \pm 23$ & $444.4 \pm 25$ & $445 \pm 24$ & $443.6 \pm 26$ & 0.09 & 0.2 \\
\hline $\mathrm{CH}$ & $8.28 \pm 0.76$ & $8.78 \pm 1.95$ & $8.28 \pm 1.38$ & $8.20 \pm 0.83$ & $8.14 \pm 1.07$ & 0.4 & 0.6 \\
\hline CRF & $5.85 \pm 1.57$ & $7.00 \pm 1.00$ & $6.85 \pm 0.89$ & $6.80 \pm 0.83$ & $6.8 \pm 0.83$ & 0.2 & 0.7 \\
\hline HOA & $4.34 \pm 0.91$ & $5.52 \pm 1.18$ & $5.37 \pm 0.94$ & $5.08 \pm 1.04$ & $4.49 \pm 1.56$ & 0.6 & 0.03 \\
\hline Coma & $3.65 \pm 2.00$ & $3.52 \pm 1.84$ & $3.32 \pm 2.01$ & $3.08 \pm 2.09$ & $2.54 \pm 1.5$ & 0.04 & 0.04 \\
\hline SA & $-0.38 \pm 0.50$ & $1.45 \pm 0.49$ & $1.92 \pm 0.63$ & $2.12 \pm 0.68$ & $2.02 \pm 0.86$ & $<0.001$ & 0.07 \\
\hline Trefoil & $1.30 \pm 0.57$ & $1.20 \pm 0.61$ & $1.11 \pm 0.60$ & $1.40 \pm 0.60$ & $0.93 \pm 0.67$ & 0.08 & 0.4 \\
\hline
\end{tabular}

Abbreviations: $1 \mathrm{~m}$, one month postoperative; $3 \mathrm{~m}, 3$ months postoperative $6 \mathrm{~m}, 6$ months postoperative; $1 \mathrm{y}$, one year postoperative; CCT, central corneal thickness; CDVA, corrected distance visual acuity; $\mathrm{CH}$, corneal hysteresis; $\mathrm{CRF}$, corneal resistance factor; D, diopters; HOA, higher order aberrations (root mean square); Km, maximum keratometry; LogMAR, logarithm of the minimum angle of resolution; Preop, preoperative; SA, spherical aberrations (root mean square); UDVA, uncorrected distance visual acuity. Values are shown as mean \pm standard deviation. $P$ values was calculated using $T$ test. 
significant change in any postoperative visits in comparison with the preoperative values.

Table 2 summarizes aberrometric outcomes of the patients after MyoRing implantation. The most significantly reduced value was primary coma. It reduced significantly 1 month after surgery $(P=0.03)$. The reduction in coma value continued during the postoperative visits. Although total higher order aberrations did not change significantly within 1 year after the surgery compared with the preoperative values, whereas it significantly reduced through the follow-up visits. Spherical aberrations significantly increased 1 month after the operation $(P<0.001)$ and trefoil reduced 1 year after the operation with a nearly significant $P$ value $(0.08)$.

According to the proposed definition for safety, ${ }^{8,9}$ this procedure was $100 \%$ safe because none of the patients lost more than two lines of Snellen CDVA. The safety index (mean postoperative CDVA/ mean preoperative CDVA) was 1.51 after 1 year, indicating 51\% increase in visual acuity (visual acuities changed to Snellen in order to calculate ratios). Efficacy (percentage of eyes achieving a UDVA of $20 / 40$ or more) of the procedure was $15 \%$. The efficacy index (mean postoperative UDVA/mean preoperative CDVA) was 1.14 in the first year, proposing that after 1 year, MyoRing alone could achieve $114 \%$ of the baseline BSCVA. Stability (percentage of eyes with less than $1 \mathrm{D}$ change in spherical equivalent from the first month to the last in 1 year) of the study was $94 \%$ (31 eyes).

No significant complication occurred during the operation and the follow-up period. Twenty-three patients $(67 \%)$ had fine corneal haziness around the implanted ring out of visual axis. One of the patients had annoying glare and was planned to have ring explantation but the patient refused to undergo the procedure.

\section{Vector analysis of refractive astigmatism}

Table 3 shows the results of Alpins vector analysis based on 1-year postoperative refractive astigmatism. SIA was significantly different from the TIA $(P=0.04)$. The DV and the IOS were not significantly different from zero (one sample $T$ test; $P=0.16$ and 0.19 , respectively). On the other hand, correction index and flattening index were not significantly different from one (one sample $T$ test; $P=0.82$ and 0.3 , respectively) (Table 3 ).

\section{Discussion}

Wedge resection along with penetrating and lamellar keratoplasty has long been available surgical options in the management of PMD. The high rate of complications in these invasive procedures, along with irreversibility and unpredictability of the results were motivations to
Table 3 Outcomes of the Alpins vector analysis

\begin{tabular}{|c|c|c|c|c|}
\hline & Mean & $S D$ & Range & $\begin{array}{l}\text { Optimal } \\
\text { value }\end{array}$ \\
\hline TIA (D) & -0.75 & 4.03 & $-7.52,+8.00$ & Same as SIA \\
\hline SIA (D) & -0.23 & 4.6 & $-7.69,+12.34$ & Same as TIA \\
\hline Difference vector (D) & -0.74 & 2.96 & $-5.5,+5.5$ & 0 \\
\hline $\begin{array}{l}\text { Angle of Difference } \\
\text { vector (degree) }\end{array}$ & -0.73 & 23.19 & $-40.05,+44.95$ & 0 \\
\hline Index of success & 0.19 & 0.84 & $-1.76,+1.28$ & 0 \\
\hline Correction index & 0.81 & 1.02 & $-1.7,+2.58$ & 1 \\
\hline Flattening effect & 0.69 & 3.9 & $-6.1,+12.24$ & \\
\hline Flattening index & 0.97 & 0.52 & $0.1,2.57$ & 1 \\
\hline
\end{tabular}

Abbreviations: D, diopters; SIA, surgically induced astigmatism; TIA, target induced astigmatism.

find new surgical options including ICRS. ${ }^{7,20-23}$ On the other hand, fitting contact lens is more difficult in PMD compared with keratoconus. ${ }^{9}$ ICRS implantation, as a minimally invasive and reversible method, is associated with faster visual rehabilitation compared with the former more invasive surgical options. ${ }^{7}$ There are reports on good safety and effectiveness profile of Intacs and KeraRing in patients with PMD. $1,7,9$ Newly proposed continuous intrastromal ring (MyoRing) has been proved to be a safe and effective procedure for keratoconus even in advanced cases. ${ }^{12-14,18,24}$

Table 4 compares the results of the current study with the previous reports on ICRS implantation in PMD. Intacs segments (Addition Technologies, CA, USA) consist of two semicircular polymethyl methacrylate segments, and each have $150^{\circ}$ arch length with a hexagonal transverse shape. External and internal diameters of each segment are 8.10 and $6.77 \mathrm{~mm}$, respectively, with variable thicknesses. The Ferrara ring segments (Mediphacos), are made of polymethyl methacrylate with a triangular crosssection to induce a prismatic effect and to reduce aberrations. KeraRings are similar to Ferrara rings, but with a wider range of available thickness and arch length. Internal and external diameters are 4.40 and $5.60 \mathrm{~mm}$, respectively. ${ }^{10}$

In the present report, implantation of MyoRing was associated with significant improvement of UDVA, and a non-significant improvement in CDVA after 1 month. Improvement in UDVA (0.49 LogMAR) is higher than the previous reports on Intacs $\left(0.17 \log \mathrm{MAR}^{18}\right.$ and 0.35 LogMAR ${ }^{9}$ ) and lower than the study conducted by Kubaloglu et al ${ }^{1}$ on KeraRing (1.05 LogMAR). On the other hand, CDVA improvement in our report is comparable with the previous reports on Intacs 9,18 and lower than the report on KeraRing. ${ }^{1}$ During the 1-year follow-up period, a non-significant improvement in both UDVA and CDVA was observed. Although the change during the follow-up period was not significant, it may be an indicator of a long-term corneal remodeling process. 
Table 4 A review over previous studies of different types of corneal intrastromal ring implantation in PMD

\begin{tabular}{|c|c|c|c|c|c|c|c|c|c|c|}
\hline Study & Ring type & year & $\begin{array}{l}\text { No. } \\
\text { of eye }\end{array}$ & $\begin{array}{l}\text { Follow-up } \\
\text { (mon) }\end{array}$ & $\begin{array}{c}\text { Change in } \\
\text { UDVA } \\
(\log M A R)\end{array}$ & $\begin{array}{c}\text { Change in } \\
\text { CDVA } \\
(\log M A R)\end{array}$ & $\begin{array}{c}\text { Change in } \\
K m \text { (D) }\end{array}$ & $\begin{array}{l}\text { Change in } \\
\text { sphere }(D)\end{array}$ & $\begin{array}{l}\text { Change in } \\
\text { cylinder }(D)\end{array}$ & $\begin{array}{l}\text { Change in } \\
\text { CCT }(\mathrm{mm})\end{array}$ \\
\hline Kubaloglu et al ${ }^{1}$ & KeraRing & 2010 & 16 & 36 & 1.05 & 0.53 & 3.62 & 2.71 & 2.01 & 8 \\
\hline Mularoni $e t a l^{7}$ & Intacs & 2005 & 8 & 12 & & & 1.49 & & 4.59 & \\
\hline Pinero et al ${ }^{18}$ & Intacs & 2009 & 21 & 6 & 0.17 & 0.21 & 1.76 & 0.01 & 2.15 & \\
\hline Ertan and Bahadri ${ }^{9}$ & Intacs & 2006 & 9 & 6 & 0.35 & 0.22 & 1.3 & 1.09 & 1.47 & \\
\hline This study & MyoRing & 2013 & 33 & 12 & 0.49 & 0.18 & 7.1 & 5.48 & 2.71 & 13.6 \\
\hline
\end{tabular}

Changes have been reported for one year postoperatively or at the end of the study if shorter. Abbreviations: D, diopter, NA, not available.

The change in the spherical part of refraction and keratometric values in this study was much higher than previous results of Intacs or KeraRing. ${ }^{1,9,18}$ More favorable outcome of KeraRing compared with Intacs ${ }^{9,23}$ in the study of Kubaloglu et al ${ }^{1}$ was probably due to extended arc length; resulting in higher arc-shortening effect, which acts like MyoRing. It can be concluded that full ring implantation has a higher arc-shortening effect compared with ring segments. ${ }^{23}$ This may show that MyoRing could be used in more advanced cases with higher myopia.

The improvement of UDVA was more significant compared with CDVA in this study and the reduction in keratometry and spherical part of the refraction was much higher than the previous reports of ICRS. 1,9,18 This may be associated with the fact that MyoRing is much more effective in flattening the cornea than in reducing the aberrations.

The reduction in keratometry and the spherical part of refraction was significant within the first month after the operation. Also, there was no significant alteration after that. This finding together with the satisfactory stability index (of 94\%) suggests the stability of the findings and probably retardation of the progressive nature of PMD after MyoRing implantation. Yet this theory needs to be assessed via long-term studies. A previous report on Intacs in keratoconus ${ }^{25}$ revealed a significant increase in the K-values 6 months after Intacs implantation. This is explained by the failure of Intacs to permanently flatten the cornea (its effect is overcome by the progression of keratoconus). The stability of the parameters in our study may be due to the difference between MyoRing and Intacs, low sample size and short follow-up period.

Mean value of cylindrical correction after 12 months was $2.71 \mathrm{D}$. This is due to central corneal flattening effect of MyoRing. ${ }^{23}$ The amount of reduction in cylindrical part of refraction is comparable with previous results of ICRS, except for the study by Mularoni, ${ }^{7}$ which shows a more significant reduction using asymmetric ICRS.

The corneal biomechanical profile (corneal hysteresis and corneal resistance factor) did not change significantly by the MyoRing implants. Insignificant increases of these parameters were also observed in the prior studies. ${ }^{24,26,27}$
This was probably because of the inferior location of thinning in PMD, which is not measured by conventional ORA. On the other hand, corneal hysteresis and corneal resistance factor may not be reliable enough to measure subtle changes. Use of the newly developed curve analysis software in ORA may be more helpful for the detection of these changes and should be addressed in future studies.

Central corneal thickness increased non-significantly after the surgery. The increase can be justified by redistribution of the stromal tissue after ring implantation. Similarly, Alio et a ${ }^{18}$ reported an increase in central corneal thickness in keratoconic patients after MyoRing implantation.

In PMD, coma aberration increases as the disease progresses. ${ }^{28}$ In the present study, coma and trefoil decreased in the final follow-up compared with the preoperative values. Pinero et al ${ }^{18}$ showed similar findings after ICRS implantation. These findings are due to regularizing effect of the ring on the corneal curvature. The spherical aberration increased significantly in the present study, which can be attributed to the central flattening effect of the MyoRing, opposed to the ICRS results $^{23}$ (insignificant reduction). This different result is most probably due to the greater flattening effect of MyoRing compared with ICRS. On the other hand, higher order aberrations did not decrease after the operation, which may be due to high increase of spherical aberrations despite the decrease in other aberrations.

To evaluate the astigmatic correcting effect of MyoRing, vector analysis of astigmatism was performed. As the amount and axis of SIA varied significantly, it was difficult to find a meaningful relationship between ring properties and astigmatism correction of our series. In calculating average in axis changes of vectors, we should consider the limitation of this value, because vectors of opposing or partly opposing directions cancel each other out in different amounts.

Vector analysis helped to determine astigmatism correction effectiveness. TIA, SIA, and DV are the three basic vectors in Alpins vector analysis. ${ }^{19}$ Other different parameters are calculated based on these 3 vectors and 
are used to determine: overcorrection or undercorrection of astigmatism (correction index), overall astigmatic correction success (IOS, flattening index), treatment misalignment (Angle of Difference vector), and residual astigmatism (flattening effect). ${ }^{29}$

Following MyoRing implantation, the DV and the IOS were not significantly different from zero and correction index and flattening index were not significantly different from one, indicating that on average, no significant overor undercorrection of astigmatism had occurred. On the other hand, the correction index was less than 1.0, indicating an overall undercorrection after MyoRing implantation. The IOS in this study was 0.19 indicating that about $81 \%$ of the astigmatic correction goal has been met.

In conclusion, a vector analysis of the refractive astigmatism changes showed that MyoRing provided a fairly effective astigmatism reduction.

We placed the arcuate incision site temporally to reduce against the rule astigmatism caused by PMD.

The limitations of this study were the unavailability of control group and limited number of cases.

In the present study, lack of serious complications in this study and high level of safety and acceptable stability and affectivity profile suggest that MyoRing is an acceptable treatment option for PMD. There are some advantages mentioned for femtosecond laser, including minimal edema of corneal stroma, lack of serious complications during the surgery, and more accurate pocket creation. ${ }^{15-17}$ Nevertheless, comparison of these two techniques needs a larger randomized controlled trial that was not possible in our study.

\section{Summary}

What was known before

- Different treatment modalities are utilized for the management of patients with PMD.

- MyoRing is considered an effective, safe, and reversible treatment modality in the vision rehabilitation of patients with corneal ectatic disorders.

What this study adds

- MyoRing may be considered as a treatment modality for spherocylindrical correction in patients with PMD, with an acceptable safety and effectiveness profile.

\section{Conflict of interest}

The authors declare no conflict of interest.

\section{References}

1 Kubaloglu A, Sari ES, Cinar Y, Koytak A, Kurnaz E, Piñero DP et al. A single 210-degree arc length intrastromal corneal ring implantation for the management of pellucid marginal corneal degeneration. Am J Ophthalmol 2010; 150(2): 185-192 e181.

2 Maguire LJ, Klyce SD, McDonald MB, Kaufman HE. Corneal topography of pellucid marginal degeneration. Ophthalmology 1987; 94(5): 519-524.

3 Rodriguez-Prats J, Galal A, Garcia-Lledo M, De La Hoz F, Alio JL. Intracorneal rings for the correction of pellucid marginal degeneration. J Cataract Refract Surg 2003; 29(7): 1421-1424.

4 Karabatsas $\mathrm{CH}$, Cook SD. Topographic analysis in pellucid marginal corneal degeneration and keratoglobus. Eye (Lond) 1996; 10(Pt 4): 451-455.

5 Sridhar MS, Mahesh S, Bansal AK, Nutheti R, Rao GN. Pellucid marginal corneal degeneration. Ophthalmology 2004; 111(6): 1102-1107.

6 Krachmer JH. Pellucid marginal corneal degeneration. Arch Ophthalmol 1978; 96(7): 1217-1221.

7 Mularoni A, Torreggiani A, di Biase A, Laffi GL, Tassinari G. Conservative treatment of early and moderate pellucid marginal degeneration: a new refractive approach with intracorneal rings. Ophthalmology 2005; 112(4): 660-666.

8 Kompella VB, Aasuri MK, Rao GN. Management of pellucid marginal corneal degeneration with rigid gas permeable contact lenses. CLAO J 2002; 28(3): 140-145.

9 Ertan A, Bahadir M. Intrastromal ring segment insertion using a femtosecond laser to correct pellucid marginal corneal degeneration. J Cataract Refract Surg 2006; 32(10): 1710-1716.

10 Pinero DP, Alio JL. Intracorneal ring segments in ectatic corneal disease - a review. Clin Experiment Ophthalmol Mar 2010; 38(2): 154-167.

11 Silvestrini TA, Mathis ML, Loomas BE, Burris TE. A geometric model to predict the change in corneal curvature from the intrastromal corneal ring (ICR). Invest Ophthalmol Vis Sci 1994; 35: 2023.

12 Daxer A, Mahmoud H, Venkateswaran RS. Intracorneal continuous ring implantation for keratoconus: One-year follow-up. J Cataract Refract Surg 2010; 36(8): 1296-1302.

13 Jabbarvand M, Salamatrad A, Hashemian H, Khodaparast M. Continuous corneal intrastromal ring implantation for treatment of keratoconus in an Iranian population. Am J Ophthalmol 2013; 155(5): 837-842.

14 Jabbarvand M, Salamatrad A, Hashemian H, Mazloumi M, Khodaparast M. Continuous intracorneal ring implantation for keratoconus using a femtosecond laser. J Cataract Refract Surg 2013; 39(7): 1081-1087.

15 Carrasquillo KG, Rand J, Talamo JH. Intacs for keratoconus and post-LASIK ectasia: mechanical versus femtosecond laser-assisted channel creation. Cornea 2007; 26(8): 956-962.

16 Rabinowitz YS, Li X, Ignacio TS, Maguen E. INTACS inserts using the femtosecond laser compared to the mechanical spreader in the treatment of keratoconus. J Refract Surg 2006; 22(8): 764-771.

17 Kanellopoulos AJ, Pe LH, Perry HD, Donnenfeld ED. Modified intracorneal ring segment implantations (INTACS) for the management of moderate to advanced keratoconus: efficacy and complications. Cornea 2006; 25(1): 29-33.

18 Pinero DP, Alio JL, Morbelli H, Uceda-Montanes A, El Kady B, Coskunseven E et al. Refractive and corneal aberrometric changes after intracorneal ring implantation in corneas with pellucid marginal degeneration. Ophthalmology 2009; 116(9): 1656-1664. 
19 Alpins NA. A new method of analyzing vectors for changes in astigmatism. J Cataract Refract Surg 1993; 19(4): 524-533.

20 Rodriguez-Gonzales-Herrero ME, Ortega AR, MoraFigueroa JM. Surgical treatment of pellucid marginal degeneration associated with cataract. J Cataract Refract Surg 2000; 26(3): 309-311.

21 Cameron JA. Results of lamellar crescentic resection for pellucid marginal corneal degeneration. Am J Ophthalmol 1992; 113(3): 296-302.

22 Kremer I, Sperber LT, Laibson PR. Pellucid marginal degeneration treated by lamellar and penetrating keratoplasty. Arch Ophthalmol 1993; 111(2): 169-170.

23 Rasheed K, Rabinowitz YS. Surgical treatment of advanced pellucid marginal degeneration. Ophthalmology 2000; 107(10): 1836-1840.

24 Alio JL, Pinero DP, Daxer A. Clinical outcomes after complete ring implantation in corneal ectasia using the femtosecond technology: a pilot study. Ophthalmology 2011; 118(7): 1282-1290
25 Alio JL, Shabayek MH, Artola A. Intracorneal ring segments for keratoconus correction: long-term follow-up. J Cataract Refract Surg 2006; 32(6): 978-985.

26 Dauwe C, Touboul D, Roberts CJ, Mahmoud AM, Kérautret $\mathrm{J}$, Fournier $\mathrm{P}$ et al. Biomechanical and morphological corneal response to placement of intrastromal corneal ring segments for keratoconus. J Cataract Refract Surg 2009; 35(10): 1761-1767.

27 Pinero DP, Alio JL, Barraquer RI, Michael R. Corneal biomechanical changes after intracorneal ring segment implantation in keratoconus. Cornea 2012; 31(5): 491-499.

28 Kamiya K, Hirohara Y, Mihashi T, Hiraoka T, Kaji Y, Oshika T. Progression of pellucid marginal degeneration and higherorder wavefront aberration of the cornea. Jpn J Ophthalmol 2003; 47(5): 523-525.

29 Visser N, Berendschot TT, Bauer NJ, Nuijts RM. Vector analysis of corneal and refractive astigmatism changes following toric pseudophakic and toric phakic IOL implantation. Invest Ophthalmol Vis Sci 2012; 53(4): 1865-1873. 